Original Paper http://ajol.info/index.php/ijbcs http://indexmedicus.afro.who.int

\title{
Le semis direct sous couverture végétale : une opportunité de mise en place rapide du cotonnier en culture pluviale au Mali
}

\author{
Fagaye SISSOKO ${ }^{1 *}$, Sidiki DIARRA ${ }^{2}$ et Mamadou TRAORE ${ }^{2}$ \\ ${ }^{1}$ Institut d'Economie Rurale (IER), Programme coton, BP 16, Sikasso, Mali. \\ ${ }^{2}$ Institut d'Economie Rurale (IER), Programme coton, BP 262, Sotuba, Mali. \\ *Auteur correspondant ; E-mail: fagaye_sissoko@yahoo.fr; Tél : +223667981 71/ + 22377033540
}

\section{REMERCIEMENTS}

Cette étude a été réalisée dans le cadre des activités du projet C4 + Togo "Renforcement technologique et diffusion des bonnes pratiques agricoles pour le coton dans les pays du Coton-4 et au Togo". Les auteurs remercient l'Etat Malien et la Coopération Brésilienne pour le financement du projet.

\section{RESUME}

En zone soudano-sahélienne, la productivité des cultures est limitée par la disponibilité de l'eau et la fertilité des sols. Le cotonnier en est particulièrement affecté lors de sa culture L'objet de cette étude est d'évaluer l'effet du semis direct sous couverture végétale sur le rendement du cotonnier. Deux variétés de cotonnier (NTA MS334 et BRS 293) ont été utilisées pour comparer 6 systèmes de culture. Le dispositif statistique utilisé a été un Split plot. Les données collectées ont concerné les échantillons de sol, les délais de levée des cotonniers, les densités, les hauteurs, les nombres de capsules et les rendements en graine. La levée a été plus rapide dans les systèmes de culture en semis sous couverture végétale. La densité à la récolte des cinq systèmes en SCV a été de 65076 plants ha ${ }^{-1}$ contre 60564 en SC. Les systèmes de culture n'ont pas eu d'effets statistiquement significatifs sur les rendements en coton graine, mais une différence significative a été observée entre les deux variétés. Le SCV est possible en zone soudano-sahélienne, la levée est plus rapide et permet d'améliorer la densité et le rendement (2 $452 \mathrm{~kg} \mathrm{ha}^{-1}$ contre $2373 \mathrm{~kg} \mathrm{ha}^{-1}$ en SC) des cultures.

(C) 2020 International Formulae Group. All rights reserved.

Mots clés : Systèmes de culture, cotonnier, densité, capsules, rendement.

\section{Direct sowing under cover crop: an opportunity for rapid establishment of rainfed cotton in Mali}

\begin{abstract}
In Sudano-Sahelian zone, crop productivity is limited by water availability and soil fertility. The purpose of this study is to evaluate the effect of direct sowing under cover crop on cotton yield. Two cotton varieties (NTA MS334 and BRS 293) were used to compare 6 cropping systems. The statistical design used was a split plot. The data collected concerned soil samples, cotton plant emergence times, densities, heights, number of bolls and cotton yields. Emergence was faster in seedling cropping systems under cover crop. Harvest density in the five SCV systems was 65076 plants ha ${ }^{-1}$ versus 60564 plants ha ${ }^{-1}$ in SC. The cropping systems had no
\end{abstract}


statistically significant effect on seed cotton yields, but a significant difference was observed between the two varieties. SCV is possible in Sudano-Sahelian zone, emergence is faster and improves crop density and yield (2 $452 \mathrm{~kg} \mathrm{ha}^{-1}$ compared to $2373 \mathrm{~kg} \mathrm{ha}^{-1}$ in SC).

(C) 2020 International Formulae Group. All rights reserved.

Keywords: Cropping systems, cotton, density, bolls, yield

\section{INTRODUCTION}

La culture du coton (Gossypium hirsutum L.) est la locomotive de l'agriculture dans la zone cotonnière du Mali. Elle joue un rôle important dans l'économie du pays (Camara, 2015). Cependant, la baisse des rendements constatée au cours des dernières décennies est attribuable à certaines contraintes (aléas climatiques, dégâts des ravageurs et dégradation des sols) avec un impact sur la sécurité alimentaire, la réduction des revenus des exploitations agricoles, l'exode rural et les conflits entre agriculteurs et éleveurs (Deveze et Hally des Fontaines, 2005).

La zone cotonnière du Mali est située entre les isohyètes 800 et $1200 \mathrm{~mm}$ (Kouressy et al., 2019). Le cotonnier est cultivé en pluvial strict, en rotation avec le maïs, le sorgho et le mil (Sissoko et al., 2015). Le cotonnier consomme environ $600 \mathrm{~mm}$ d'eau durant son cycle de développement (Cetin et Bilgel, 2002). Le cumul pluviométrique enregistré pendant l'année est très souvent supérieur aux besoins en eau de cette culture. Il n'est pas un bon indicateur de l'eau disponible pour la culture du cotonnier. La distribution spatiotemporelle des pluies qui est un élément essentiel pour la croissance et la productivité des plantes cultivées est souvent très irrégulière.

Le semis direct sous couverture végétale est une pratique de l'agriculture de conservation (AC) basée sur une gestion intégrée des sols, de l'eau et des ressources biologiques combinées à l'utilisation des herbicides et des engrais minéraux (Giller et al., 2015). Elle est fondée sur trois principes qui sont censés améliorer les processus biologiques en surface et en profondeur du sol. Celles-ci sont : (1) perturbation mécanique minimale ou nulle du sol ; (2) couverture organique permanente du sol (constituée d'une culture en croissance ou d'un paillis mort de résidus de culture) et (3) rotations des cultures.

Dans la pratique, les agriculteurs n'adoptent pas tous les principes de l'AC pour diverses causes, telles que l'accès limité aux intrants (herbicides, semences de cultures de couverture), les contraintes de main-d'œuvre ou les ressources pour cultiver des cultures de rente (Baudron et al., 2007). Dans les conditions du Mali, c'est surtout la gestion des résidus de récolte qui pose problème avec la divagation des animaux pendant la saison sèche.

Dans les régions semi-arides, le paillage s'est révélé efficace pour réduire le risque de mauvaises récoltes au niveau des champs grâce à une meilleure captation et utilisation des précipitations (Scopel et al., 2004 ; Bationo et al., 2007). Dans les semis sous couverture végétale (SCV) pratiqués au Mali, la quantité de biomasse disponible dans les parcelles varie entre 3,5 et 7,4 tonnes. ha ${ }^{-1}$. Cette importante biomasse permet d'enrichir le stock de matière organique $(\mathrm{MO})$ du sol et contribue à la rétention de l'humidité du sol (Turnel et al., 2015). La présence d'un couvert végétal avant le semis de la culture, stimule l'activité biologique du sol en créant des habitats pour les auxiliaires. Enfin, la couverture végétale protège le sol contre les aléas climatiques en limitant les phénomènes d'érosion, de ruissellement et de battance des sols (Naudin et al., 2010 ; Sissoko et al., 2013). Ainsi, le SCV est considéré comme une solution de rechange à l'agriculture conventionnelle qui pratique le travail du sol (Gowing et Palmer, 2008). Dans le cadre d'une série d'expériences menées dans des zones subhumides, semi-arides et subhumides sèches d'Afrique orientale, Rockstrom et al. (2008), ont montré que les pratiques de travail minimal du sol permettaient d'accroître le rendement des 
cultures, même lorsque le paillage des résidus végétaux était faible ou nul.

Concernant la dégradation des sols, les causes majeures sont entre autres, le rythme élevé de la croissance démographique amplifiant la pression sur les terres arables et les terres marginales ainsi que l'extension annuelle des superficies cultivées et la réduction des pâturages et des friches (Odru, 2013 ; Fleury, 2016). En zone cotonnière du Mali, les superficies exploitées en culture cotonnière sont passées de 28360 ha en 1960 à 698158 ha en 2018, soit une augmentation moyenne annuelle de 40,72\% (Traoré, 2018).

Pour la restauration de la fertilité des sols, les systèmes de culture en zone cotonnière se caractérisaient dans un passé récent, par un temps de mise en culture de trois à cinq ans, alternant avec un temps de jachère qui dépassait généralement dix ans, pour permettre la restauration naturelle du couvert végétal et de la fertilité des sols (Fairhurst, 2015). De nos jours, le système de jachère de longue durée n'est plus possible avec la pression démographique.

Pour couvrir les besoins d'une population en pleine croissance, des alternatives de gestion durable de la fertilité des sols et de l'eau ont été développées et introduites en milieu réel parmi lesquelles, le SCV. Cette technique utilisée au Brésil permet de diminuer l'effet de l'érosion éolienne et hydrique et d'améliorer les rendements (Scopel, 2004). L'objectif de cette étude a été d'évaluer l'effet du semis direct sous couverture végétale sur le rendement du cotonnier.

\section{MATERIEL ET METHODES}

\section{Site d'étude}

L'expérimentation a été conduite de 2014 à 2018 au Centre Régional de Recherche Agronomique (CRRA) de SOTUBA situé dans la commune I du district de Bamako avec une latitude de $12^{\circ} 66^{\prime} 38^{\prime \prime}$ Nord et une longitude de 7'92'22" Ouest sur une altitude de $320 \mathrm{~m}$. Le climat est de type soudano sahélien avec deux saisons (une saison pluvieuse de juin à octobre et une saison sèche de novembre à mai).
Le cumul de la pluviométrie moyenne des dix dernières années a été de $920 \mathrm{~mm}$ en 70 jours de pluie, tandis que celui de la période de l'expérimentation (2014-2018) a été de 838,6 $\mathrm{mm}$ en 71 jours de pluie (Tableau 1).

\section{Matériel}

Pour la réalisation de cette expérimentation, deux variétés de cotonnier à haut potentiel de rendement ont été cultivées (BRS 293 et NTA MS334). La variété BRS 293 est originaire du Brésil et a été introduite au Mali en 2009 tandis que la variété NTA MS334 est une création du programme coton de l'Institut d'Economie Rurale (IER) du Mali. Quelques caractéristiques morphophysiologiques, agronomiques et technologiques des deux variétés figurent dans le Tableau 2. La fertilisation minérale du cotonnier a été faite avec l'engrais coton ( $14 \mathrm{~N}$ $\left.18 \mathrm{P}_{2} \mathrm{O}_{5}-18 \mathrm{~K}_{2} \mathrm{O}+6 \mathrm{~S}+1 \mathrm{~B}_{2} \mathrm{O}_{3}\right)$ et l'urée $(46 \%$ de $\mathrm{N})$. La protection phytosanitaire du cotonnier a été faite avec les matières actives Emamectine Benzoate 19,2 g/l en Emulsion Concentrée (EC) pour les deux premiers traitements insecticides et Cyperméthrine $72 \mathrm{~g} / \mathrm{l}+$ Acétamipride $32 \mathrm{~g} / \mathrm{l}$ (EC) pour les quatre derniers traitements.

\section{Méthodes}

\section{Dispositif expérimental}

Le dispositif expérimental a été un Split-plot avec les systèmes de culture comme facteur principal et les variétés en facteur secondaire, le tout répété 4 fois. Les systèmes de culture comparés comportaient les 6 niveaux suivants : $\mathrm{T} 1=$ résidus de maïs $; \mathrm{T} 2=$ résidus de maïs + Brachiaria ruziziensis; $\mathrm{T} 3$ $=$ résidus de maïs + Brachiaria ruziziensis + Stylosanthes hamata $; \mathrm{T} 4=$ résidus de maïs + Brachiaria ruziziensis + Cajanus cajan ; T5 = résidus de maïs + Brachiaria ruziziensis + Mucuna cochinchinensis; $\mathrm{T} 6=$ système conventionnel, exportation des résidus après la récolte. Deux variétés ont été utilisées comme facteur secondaire : V1 : NTA MS334 et V2 : BRS 293. La superficie totale de l'essai a été de $4227,4 \mathrm{~m}^{2}$, soit $91,9 \mathrm{~m} \times 46 \mathrm{~m}$. La superficie des parcelles élémentaires a été de $144 \mathrm{~m}^{2}$ (18 lignes de semis), soit un écartement 
de $14,4 \mathrm{~m} \times 10 \mathrm{~m}$. Les graines de coton ont été semées dans des poquets de trois centimètres de profondeur aux écartements de $0,80 \mathrm{~m}$ entre les lignes de semis et $0,30 \mathrm{~m}$ entre les poquets sur la ligne de semis. Le démariage a été réalisé à 2 plants par poquet 15 jours après le semis. Trois sarclages et un buttage ont été réalisés dans les parcelles du système conventionnel (T6) au cours du cycle de développement végétatif des cotonniers. Des petites raies ont été tracées le long de la ligne de semis. L'engrais coton $\left(14 \mathrm{~N}-18 \mathrm{P}_{2} \mathrm{O}_{5}\right.$ $\left.18 \mathrm{~K}_{2} \mathrm{O}+6 \mathrm{~S}+1 \mathrm{~B}_{2} \mathrm{O}_{3}\right)$ a été appliqué 14 jours après la levée (JAL) des cotonniers dans des raies en ligne continue puis minutieusement refermées. L'engrais coton a été apporté à la dose de $200 \mathrm{~kg} \cdot \mathrm{ha}^{-1}$ dans toutes les parcelles élémentaires. Dans les parcelles des systèmes de culture avec résidus de récolte en couverture, un apport supplémentaire de $50 \mathrm{~kg}$ d'urée ha ${ }^{-1}$ a été fait au moment de l'apport de l'engrais coton. Au 45 $\mathrm{JAL}$, un apport complémentaire d'urée de $50 \mathrm{~kg} \cdot \mathrm{ha}^{-1}$ a été fait dans toutes les parcelles élémentaires.

\section{Échantillonnage du sol et analyse physico- chimique}

Trois fosses pédologiques de $1,5 \mathrm{~m}$ de profondeur, $1,5 \mathrm{~m}$ de longueur et $0,80 \mathrm{~m}$ de largeur ont été ouvertes sur une superficie d'un hectare pour la description du profil pédologique en 2014. Trois points de prélèvement de sol à la tarière ont été faits dans chacun des quatre répétitions de l'essai et un échantillon composite a été constitué pour les profondeurs $0-20 \mathrm{~cm}$ et $20-40 \mathrm{~cm}$ en 2014 . Huit échantillons ont été envoyés au laboratoire de Sotuba pour des analyses physiques et chimiques: $\mathrm{pH}$ eau, azote totale $(\mathrm{N})$, phosphore total et assimilable $(\mathrm{P})$, potassium assimilable $(\mathrm{K})$, calcium $(\mathrm{Ca})$, magnésium (Mg) et capacité d'échange cationique CEC).

\section{Collecte des données}

La date à laquelle $50 \%$ des poquets théoriques ont levé a été notée. Cette date a été déterminée par parcelle élémentaire en passant tous les jours après l'apparition de la première plantule. Le comptage a été réalisé sur 20 poquets successifs sur les 4 lignes centrales. Les estimations de densités de peuplement ont été effectuées sur les 4 lignes centrales de chaque parcelle élémentaire après le démariage et à la récolte. Les hauteurs de cotonniers ont été estimées sur vingt (20) plants dans chaque parcelle élémentaire à la récolte. Le nombre de capsules a été déterminé sur 5 plants dans chaque parcelle élémentaire. Le rendement a été déterminé à partir du poids de coton graine récolté sur les quatre lignes centrales de chaque parcelle élémentaire.

\section{Analyse statistique}

L'analyse de variance a été faite à l'aide du logiciel StatBox 6.5 et le test de Newman et Keuls au seuil de $5 \%$ pour la comparaison des moyennes.

Tableau 1 : Dates de semis, quantités de pluie enregistrées au mois de juin, cumul annuel et nombre de jours de pluie à Sotuba de 2014 à 2018.

\begin{tabular}{lcccccc}
\hline Années & $\begin{array}{c}\text { Dates de } \\
\text { semis }\end{array}$ & \multicolumn{3}{c}{ Mois de juin } & $\begin{array}{c}\text { Cumul } \\
\text { annuel }\end{array}$ & $\begin{array}{c}\text { Nombre de } \\
\text { jours de pluies }\end{array}$ \\
\cline { 3 - 5 } & & $\mathbf{1}^{\mathbf{e}}$ décade & $\mathbf{2}^{\mathbf{e}}$ décade & $\mathbf{3}^{\mathbf{e}}$ décade & & jours \\
\hline 2014 & 19 juin & 117,1 & 35 & 60,0 & 881,6 & 69 \\
2015 & 13 juin & 12,1 & 58,3 & 0,0 & 782,7 & 75 \\
2016 & 23 juin & 49,0 & 15,0 & 103,5 & 833,5 & 76 \\
2017 & 08 juin & 74,8 & 10,2 & 47,7 & 857,6 & 59 \\
2018 & 01 juin & 19,1 & 31,1 & 42,2 & 837,8 & 78 \\
\hline
\end{tabular}


Tableau 2 : Caractéristiques morpho-physiologiques, agronomiques et technologiques des deux variétés de cotonnier utilisées dans l'expérimentation à Sotuba de 2014 à 2018.

\begin{tabular}{lcc}
\hline Caractéristique & NTA MS 334 & BRS 293 \\
\hline Morpho-physiologiques & & \\
Nombre moyen de BV & 1,7 & 1,9 \\
Hauteur (cm) & 129,6 & 124,9 \\
\hline Agronomiques & & \\
Rendement coton graine (kg/ha) & 1504,9 & 1731,0 \\
Rendement égrenage (\%F sc.) & 43,2 & 43,0 \\
PMC (g) & 3,9 & 4,0 \\
Seed index (g) & 8,2 & 7,6 \\
\hline Technologie fibre (CERFITEX, Ségou) & 28,7 & 28,9 \\
Longueur (mm) & 81,7 & 82,3 \\
Uniformité (\%) & 4,2 & 3,8 \\
Indice micronaire & 90 & 90 \\
Maturité (\%) & 28,6 & 29,1 \\
Ténacité (g/Tex) & 5,6 & 6,0 \\
Allongement (\%) & 76,4 & 77,4 \\
Réflectance (\%) & 9,8 & 9,6 \\
Indice de Jaune (+b) & &
\end{tabular}

$\mathrm{BV}$ : branche végétative, $\mathrm{PMC}$ : poids moyen capsulaire, Seed index : indice de semences

\section{RESULTATS}

\section{Type de sol et caractéristiques physico- chimiques des sols}

L'examen du profil pédologique a permis de découvrir une succession de couches expliquant la formation et l'évolution du sol qui a été classé comme sol ferrugineux tropical avec une hydromorphie de profondeur. L'analyse granulométrique effectuée a montré qu'en surface $(0-20 \mathrm{~cm})$ le sol est sablolimoneux avec 14,6 g/100g d'argile, 37,4 $\mathrm{g} / 100 \mathrm{~g}$ de limon et $47,9 \mathrm{~g} / 100 \mathrm{~g}$ de sable. En profondeur $(20-40 \mathrm{~cm})$, il est limoneux avec $19,4 \mathrm{~g} / 100 / \mathrm{g}$ d'argile, $39,3 \mathrm{~g} / 100 \mathrm{~g}$ de limon et $40,6 \mathrm{~g} / 100 \mathrm{~g}$ de sable. Le $\mathrm{pH}$ eau moyen de la couche $0-20 \mathrm{~cm}$ a été de 6,1 tandis que dans la couche $20-40 \mathrm{~cm}$, il a été de 6,4 (Tableau 3). Ces $\mathrm{pH}$ sont légèrement acides $(6<\mathrm{pH}<6,6)$. La teneur en azote total était faible aussi bien en surface $(0,03 \mathrm{~g} / 100 \mathrm{~g})$ qu'en profondeur $(0,02 \mathrm{~g} / 100 \mathrm{~g}) . \quad \mathrm{La}$ capacité d'échange cationique était faible $5,1 \mathrm{Cmol}^{+} / \mathrm{kg}$ dans l'horizon $0-20 \mathrm{~cm}$ et $5,7 \mathrm{Cmol}^{+} / \mathrm{kg}$ dans l'horizon $20-40 \mathrm{~cm}$.

Effet du système de culture, de la variété et de l'année sur le nombre de jours entre le semis et la levée des cotonniers

Le nombre moyen de jours entre le semis et la levée a varié entre 3,8 et 9,0 jours. L'analyse statistique a montré un effet significatif entre les systèmes de culture sur le nombre de jours entre le semis et la levée en 2014. Deux groupes homogènes ont été obtenus. Le premier groupe est constitué par les systèmes de culture T1 et T6 (Tableau 4). Le second groupe est composé des autres systèmes de culture (T2, T3, T4 et T5) avec un nombre moyen de jours variant entre 3,8 et 4,6 entre le semis et la levée. Au cours de la même année, l'analyse statistique a montré une différence entre les deux variétés. La levée a été plus rapide dans les parcelles avec la BRS 293 (Tableau 4).

En 2015, l'analyse statistique a montré un effet significatif entre les systèmes de culture, cinq groupes homogènes ont été obtenus. Aucune différence n'a été observée entre les variétés.

Pendant les cinq années d'expérimentation, aucune différence significative n'a été observée entre l'interaction systèmes de culture et variétés (Tableau 4).

Effet du système de culture, de la variété et de l'année sur la densité des cotonniers après le démariage

Pendant les cinq années d'expérimentation (2014-2018), la densité moyenne dans les systèmes de culture a varié entre 54398 et 81771 plants ha $^{-1}$. C'est seulement en 2018 que l'analyse statistique a montré une différence significative $(p=0,000)$ entre les systèmes de culture sur le nombre de plants ha ${ }^{-1}$ après le démariage. Quatre groupes homogènes ont été obtenus et la plus faible 
densité a été observée dans les parcelles du système de culture T6 (Tableau 5).

A part l'année 2017, une différence significative a été observée entre les variétés sur le nombre de plants ha $^{-1}$. Les meilleures densités ont généralement été observées dans les systèmes de culture avec la variété BRS 293 (Tableau 5). Pendant les cinq années d'expérimentation, aucune interaction n'a été significative.

Effet du système de culture, de la variété et de l'année sur la densité des cotonniers à la récolte

Les systèmes de culture comparés ont eu des effets statistiquement significatifs sur le nombre de plantes ha $^{-1}$ à la récolte en $2015(p$ $=0,007)$ et en $2018(p=0,005)$ (Tableau 6). Les plus faibles densités ont été obtenues en 2015 dans les parcelles du système de culture T1 qui ont été statistiquement équivalentes aux densités des systèmes de culture T4 et T6. En 2018, les plus faibles densités ont également été obtenues dans les parcelles du système de culture T6 qui ont été statistiquement équivalentes aux densités du système de culture du T1 (Tableau 6). Les interactions (Systèmes de culture $\mathrm{x}$ variétés) n'ont pas été significatives sur le nombre de plants ha $^{-1}$ à la récolte.

A l'exemption de l'année 2018, une baisse moyenne de 4 à $8 \%$ du nombre de plants ha $^{-1}$ a été constatée dans les parcelles de tous les systèmes de culture entre le démariage et la récolte.

Effets du système de culture, de la variété et de l'année sur les hauteurs des cotonniers

$\mathrm{Au}$ cours des cinq années d'expérimentation, la hauteur des cotonniers a varié entre 90 et $150 \mathrm{~cm}$. A part l'année 2016, les systèmes de culture n'ont pas eu d'effets significatifs sur la croissance des plantes, comme l'indique les valeurs moyennes obtenues (Tableau 7).
Une différence significative a été observée entre des deux variétés sur la hauteur des cotonniers, sauf en 2015 (Tableau 7). En moyenne, les cotonniers de la variété NTA MS334 ont été plus grands que ceux de la variété BRS 293.

Aucune interaction (systèmes de culture $x$ variétés) n'a été significative pendant les cinq années d'expérimentation (Tableau 7).

Effet du système de culture, de la variété et de l'année sur le nombre de capsules par plant

Le nombre moyen de capsules par plant a varié entre 9,70 et 22,75 (Tableau 8). L'analyse statistique n'a pas montré de différence significative entre les systèmes de culture sur le nombre de capsules par plant (Tableau 8). Sauf en 2014, des différences significatives ont été observées entre les variétés sur le nombre de capsules par plants. Le nombre élevé de capsules a toujours été obtenu avec la variété BRS 293. E, 2014, le nombre moyen le plus élevé de capsules par plant $(18,61)$ a été observé.

Aucune interaction (Systèmes de culture $\mathrm{x}$ variétés) n'a été significative (Tableau 8).

Effet du système de culture, de la variété et de l'année sur les rendements obtenus

Aucune différence significative n'a été obtenue entre les systèmes de culture sur le rendement (Tableau 9). Cependant, une différence significative a été obtenue entre les deux variétés sur le rendement en coton graine au cours de chacune des années. Les meilleurs rendements ont toujours été obtenus avec la variété BRS 293. Les écarts de rendement entre la variété NTA MS334 et la variété BRS ont varié entre 251 et $629 \mathrm{~kg} \mathrm{ha}^{-1}$ en fonction des années (Tableau 9).

Tableau 3 : Caractéristiques chimiques des échantillons de sol prélevés à Sotuba en 2014.

\begin{tabular}{|c|c|c|c|c|c|c|c|c|}
\hline \multirow[t]{2}{*}{ Couches $(\mathrm{cm})$} & \multirow[t]{2}{*}{ pH eau } & \multirow{2}{*}{$\begin{array}{c}\mathrm{N} \\
\mathrm{g} / 100 \mathrm{~g}\end{array}$} & $P$ total & P ass & $\mathrm{Ca}^{++}$ & $\mathbf{M g}^{++}$ & $\mathbf{K}^{+}$ & CEC \\
\hline & & & & $\mathrm{mg} / \mathrm{kg}$ & & \multicolumn{3}{|c|}{$\mathrm{cmol}^{+} / \mathrm{kg}$} \\
\hline $0-20$ & 6,1 & 0,03 & 121,2 & 4,3 & 2,0 & 0,9 & 0,20 & 5,1 \\
\hline $20-40$ & 6,4 & 0,02 & 113,5 & 1,2 & 2,5 & 1,1 & 0,10 & 5,7 \\
\hline
\end{tabular}




\section{F. SISSOKO et al. / Int. J. Biol. Chem. Sci. 14(3): 722-738, 2020}

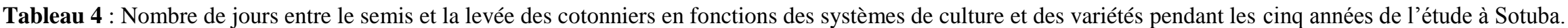

\begin{tabular}{|c|c|c|c|c|c|c|c|c|c|c|c|c|c|c|c|}
\hline \multirow{2}{*}{$\begin{array}{l}\text { Système de } \\
\text { culture }\end{array}$} & \multicolumn{3}{|c|}{2014} & \multicolumn{3}{|c|}{2015} & \multicolumn{3}{|c|}{2016} & \multicolumn{3}{|c|}{2017} & \multicolumn{3}{|c|}{2018} \\
\hline & $\begin{array}{l}\text { NTA } \\
\text { MS334 }\end{array}$ & $\begin{array}{l}\text { BRS } \\
293\end{array}$ & Moy & $\begin{array}{l}\text { NTA } \\
\text { MS334 }\end{array}$ & $\begin{array}{l}\text { BRS } \\
293\end{array}$ & Moy & $\begin{array}{l}\text { NTA } \\
\text { MS334 }\end{array}$ & $\begin{array}{l}\text { BRS } \\
293\end{array}$ & Мoy & $\begin{array}{l}\text { NTA } \\
\text { MS334 }\end{array}$ & $\begin{array}{l}\text { BRS } \\
293\end{array}$ & Moy & $\begin{array}{l}\text { NTA } \\
\text { MS334 }\end{array}$ & $\begin{array}{l}\text { BRS } \\
293\end{array}$ & Моy \\
\hline $\mathrm{T} 1$ & 7,5 & 6,3 & $6,9 a$ & 9,0 & 7,8 & $8,4 \mathrm{a}$ & 7,0 & 7,0 & $7,0 \mathrm{a}$ & 6,3 & 6,3 & $6,3 \mathrm{a}$ & 7,5 & 6,5 & $7,0 \mathrm{a}$ \\
\hline $\mathrm{T} 2$ & 4,5 & 4,0 & $4,3 \mathrm{~b}$ & 5,5 & 5,0 & $5,3 \mathrm{c}$ & 4,0 & 4,0 & $4,0 \mathrm{~b}$ & 4,0 & 4,0 & $4,0 \mathrm{~b}$ & 4,0 & 4,0 & $4,0 \mathrm{~b}$ \\
\hline T3 & 3,8 & 3,8 & $3,8 \mathrm{~b}$ & 4,0 & 4,0 & $4,0 \mathrm{~d}$ & 4,3 & 4,3 & $4,3 \mathrm{~b}$ & 4,0 & 4,0 & $4,0 \mathrm{~b}$ & 4,5 & 4,5 & $4,5 \mathrm{~b}$ \\
\hline $\mathrm{T} 4$ & 4,8 & 4,5 & $4,6 \mathrm{~b}$ & 4,0 & 4,0 & $4,0 \mathrm{~d}$ & 4,0 & 4,0 & $4,0 \mathrm{~b}$ & 4,0 & 4,0 & $4,0 \mathrm{~b}$ & 4,3 & 4,3 & $4,3 \mathrm{~b}$ \\
\hline T5 & 4,3 & 4,0 & $4,1 \mathrm{~b}$ & 4,5 & 4,5 & $4,5 \mathrm{~cd}$ & 4,3 & 4,3 & $4,3 \mathrm{~b}$ & 4,3 & 4,3 & $4,3 \mathrm{~b}$ & 4,8 & 4,8 & $4,8 \mathrm{~b}$ \\
\hline T6 & 7,8 & 6,8 & $7,3 \mathrm{a}$ & 7,8 & 6,5 & $7,1 \mathrm{~b}$ & 7,3 & 6,8 & $7,0 \mathrm{a}$ & 7,0 & 6,5 & $6,8 \mathrm{a}$ & 7,8 & 7,3 & $7,5 \mathrm{a}$ \\
\hline Moyenne & $5,4 \mathrm{a}$ & $4,8 \mathrm{~b}$ & 5,1 & 5,8 & 5,3 & 5,5 & 5,1 & 5,0 & 5,1 & 4,9 & 4,8 & 4,9 & 5,5 & 5,2 & 5,3 \\
\hline $\begin{array}{l}\text { Probabilité } \\
\text { traitement }\end{array}$ & & & 0,000 & & & 0,000 & & & 0,000 & & & 0,000 & & & 0,000 \\
\hline $\begin{array}{l}\text { Probabilité } \\
\text { variété }\end{array}$ & & & 0,008 & & & 0,056 & & & 0,484 & & & 0,589 & & & 0,160 \\
\hline $\begin{array}{l}\text { Probabilité } \\
\text { interaction }\end{array}$ & & & 0,402 & & & 0,456 & & & 0,764 & & & 0,904 & & & 0,464 \\
\hline Ecart type & & & 0,67 & & & 0,88 & & & 0,40 & & & 0,52 & & & 0,61 \\
\hline $\mathrm{CV}(\%)$ & & & 12,96 & & & 15,94 & & & 7,91 & & & 10,66 & & & 11,39 \\
\hline
\end{tabular}

$\mathrm{T} 1=$ résidus de maïs (conservation des résidus sur la parcelle après la récolte) $; \mathrm{T} 2=$ résidus de maïs + Brachiaria ruziziensis $; \mathrm{T} 3=$ résidus de maïs $+\mathrm{Brachiaria}$ ruziziensis + Stylosanthes hamata $; \mathrm{T} 4=$ résidus de maïs + Brachiaria ruziziensis + Cajanus cajan $; \mathrm{T} 5=$ résidus de maïs + Brachiaria ruziziensis + Mucuna cochinchinensis $; \mathrm{T} 6=$ système conventionnel, exportation des résidus après la récolte

Signification : les chiffres suivis des mêmes lettres ne sont pas statistiquement différents ; CV\% : Coefficient de Variation en pourcentage ; Moy = moyenne. 
F. SISSOKO et al. / Int. J. Biol. Chem. Sci. 14(3): 722-738, 2020

Tableau 5: Nombre de plants après le démariage en fonctions des systèmes de culture et des variétés pendant les cinq années de l'étude à Sotuba.

\begin{tabular}{|c|c|c|c|c|c|c|c|c|c|c|c|c|c|c|c|}
\hline \multirow{2}{*}{$\begin{array}{l}\text { Système } \\
\text { de culture }\end{array}$} & \multicolumn{3}{|c|}{2014} & \multicolumn{3}{|c|}{2015} & \multicolumn{3}{|c|}{2016} & \multicolumn{3}{|c|}{2017} & \multicolumn{3}{|c|}{2018} \\
\hline & $\begin{array}{c}\text { NTA } \\
\text { MS334 } \\
\end{array}$ & $\begin{array}{c}\text { BRS } \\
293 \\
\end{array}$ & Moy & $\begin{array}{c}\text { NTA } \\
\text { MS334 } \\
\end{array}$ & $\begin{array}{c}\text { BRS } \\
293 \\
\end{array}$ & Moy & $\begin{array}{c}\text { NTA } \\
\text { MS334 }\end{array}$ & $\begin{array}{c}\text { BRS } \\
293 \\
\end{array}$ & Moy & $\begin{array}{c}\text { NTA } \\
\text { MS334 } \\
\end{array}$ & $\begin{array}{c}\text { BRS } \\
293 \\
\end{array}$ & Moy & $\begin{array}{c}\text { NTA } \\
\text { MS334 } \\
\end{array}$ & BRS 293 & Moy \\
\hline $\mathrm{T} 1$ & 64294 & 58565 & 61429 & 62083 & 79271 & 70677 & 71150 & 73734 & 72442 & 67509 & 66314 & 66912 & 62731 & 68866 & $65799 \mathrm{~b}$ \\
\hline $\mathrm{T} 2$ & 67882 & 58507 & 63194 & 72396 & 80208 & 76302 & 65506 & 70200 & 67853 & 66498 & 65028 & 65763 & 75868 & 79398 & $77633 \mathrm{a}$ \\
\hline $\mathrm{T} 3$ & 73332 & 63252 & 68287 & 74375 & 81562 & 77969 & 65032 & 71677 & 68354 & 64108 & 64706 & 64407 & 71933 & 74132 & $73032 \mathrm{ab}$ \\
\hline $\mathrm{T} 4$ & 69792 & 65741 & 67766 & 68958 & 80000 & 74479 & 62975 & 72943 & 67959 & 63879 & 64614 & 64246 & 68692 & 73264 & $70978 \mathrm{ab}$ \\
\hline $\mathrm{T} 5$ & 71007 & 61921 & 66464 & 72292 & 78958 & 75625 & 68460 & 73892 & 71176 & 64844 & 64844 & 64844 & 72049 & 74074 & $73061 \mathrm{ab}$ \\
\hline $\mathrm{T} 6$ & 61863 & 56829 & 59346 & 64062 & 81771 & 72917 & 68513 & 72627 & 70570 & 65625 & 62684 & 64154 & 54398 & 64583 & $59491 \mathrm{c}$ \\
\hline Moyenne & 68027 a & $60802 \mathrm{~b}$ & 64415 & $69028 \mathrm{~b}$ & 80295 a & 74661 & $66939 \mathrm{~b}$ & 72512 a & 69726 & 65411 & 64698 & 65055 & $67612 \mathrm{~b}$ & 72386 a & 69999 \\
\hline $\begin{array}{l}\text { Probabilité } \\
\text { traitement }\end{array}$ & & & 0,136 & & & 0,086 & & & 0,727 & & & 0,931 & & & 0,000 \\
\hline $\begin{array}{c}\text { Probabilité } \\
\text { variété }\end{array}$ & & & 0,003 & & & 0,001 & & & 0,012 & & & 0,494 & & & 0,000 \\
\hline $\begin{array}{l}\text { Probabilité } \\
\text { interaction }\end{array}$ & & & 0,948 & & & 0,103 & & & 0,938 & & & 0,886 & & & 0,107 \\
\hline Ecart type & & & 3647 & & & 2589 & & & 1934 & & & 1084 & & & 6417 \\
\hline $\mathrm{CV}(\%)$ & & & 11,9 & & & 6,70 & & & 10,42 & & & 9,40 & & & 8,13 \\
\hline
\end{tabular}

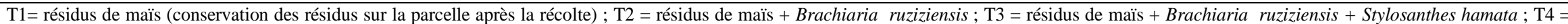

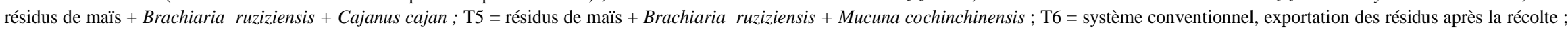
Signification : les chiffres suivis des mêmes lettres ne sont pas statistiquement différents $; \mathrm{CV} \%$ : Coefficient de Variation en pourcentage $;$ Moy $=$ moyenne. 
F. SISSOKO et al. / Int. J. Biol. Chem. Sci. 14(3): 722-738, 2020

Tableau 6: Nombre de plants à la récolte en fonctions des systèmes de culture et des variétés pendant les cinq années de l'étude à Sotuba.

\begin{tabular}{|c|c|c|c|c|c|c|c|c|c|c|c|c|c|c|c|}
\hline \multirow{2}{*}{$\begin{array}{l}\text { Systèmes } \\
\text { de culture }\end{array}$} & \multicolumn{3}{|c|}{2014} & \multicolumn{3}{|c|}{2015} & \multicolumn{3}{|c|}{2016} & \multicolumn{3}{|c|}{2017} & \multicolumn{3}{|c|}{2018} \\
\hline & $\begin{array}{l}\text { NTA } \\
\text { MS334 }\end{array}$ & BRS 293 & Moy & $\begin{array}{l}\text { NTA } \\
\text { MS334 }\end{array}$ & BRS 293 & Moy & $\begin{array}{l}\text { NTA } \\
\text { MS334 }\end{array}$ & BRS 293 & Moy & $\begin{array}{l}\text { NTA } \\
\text { MS334 }\end{array}$ & $\begin{array}{l}\text { BRS } \\
293\end{array}$ & Moy & $\begin{array}{l}\text { NTA } \\
\text { MS334 }\end{array}$ & $\begin{array}{l}\text { BRS } \\
293\end{array}$ & Moy \\
\hline $\mathrm{T} 1$ & 60185 & 54919 & 57552 & 56875 & 73333 & $65104 \mathrm{c}$ & 62869 & 69146 & 66007 & 65349 & 63557 & 64453 & 65683 & 68634 & $\begin{array}{c}67159 \\
\text { ab }\end{array}$ \\
\hline $\mathrm{T} 2$ & 62442 & 54109 & 58275 & 68958 & 74375 & $71667 \mathrm{ab}$ & 59072 & 64346 & 61709 & 64430 & 62730 & 63580 & 74132 & 75926 & $75029 \mathrm{a}$ \\
\hline $\mathrm{T} 3$ & 66782 & 58507 & 62645 & 69687 & 76563 & 73125 a & 59072 & 67827 & 63449 & 61719 & 62086 & 61903 & 74884 & 74363 & $74624 \mathrm{a}$ \\
\hline $\mathrm{T} 4$ & 66551 & 61400 & 63976 & 61771 & 73750 & $67760 \mathrm{bc}$ & 58597 & 68143 & 63370 & 61259 & 61581 & 61420 & 72396 & 73843 & $73119 a$ \\
\hline T5 & 65799 & 59028 & 62413 & 66042 & 74792 & $70417 \mathrm{ab}$ & 61814 & 69884 & 65849 & 62408 & 62546 & 62477 & 72338 & 72685 & $72512 \mathrm{a}$ \\
\hline T6 & 57870 & 53183 & 55527 & 58854 & 75521 & $67187 \mathrm{bc}$ & 61762 & 70200 & 65981 & 58869 & 59421 & 59145 & 61111 & 62095 & $61603 \mathrm{~b}$ \\
\hline Moyenne & $63272 \mathrm{a}$ & $56858 \mathrm{~b}$ & 60065 & $63698 \mathrm{~b}$ & $74722 \mathrm{a}$ & 69210 & $60531 \mathrm{~b}$ & $68258 \mathrm{a}$ & 64394 & 62339 & 61987 & 62163 & 70090 & 71259 & 70674 \\
\hline $\begin{array}{l}\text { Probabilité } \\
\text { traitement }\end{array}$ & & & 0,163 & & & 0,007 & & & 0,731 & & & 0,396 & & & 0,005 \\
\hline $\begin{array}{l}\text { Probabilité } \\
\text { variété }\end{array}$ & & & 0,005 & & & 0,001 & & & 0,001 & & & 0,593 & & & 0,379 \\
\hline $\begin{array}{l}\text { Probabilité } \\
\text { interaction }\end{array}$ & & & 0,992 & & & 0,051 & & & 0,987 & & & 0,783 & & & 0,377 \\
\hline Ecart type & & & 4535 & & & 7795 & & & 6841 & & & 4960 & & & 6421 \\
\hline $\mathrm{CV}(\%)$ & & & 12,30 & & & 4,60 & & & 10,62 & & & 7,98 & & & 9,09 \\
\hline
\end{tabular}

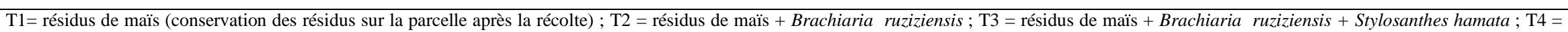

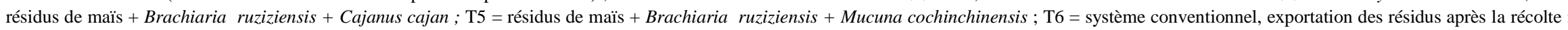
Signification : les chiffres suivis des mêmes lettres ne sont pas statistiquement différents ; CV\% : Coefficient de Variation en pourcentage ; Moy $=$ moyenne. 
F. SISSOKO et al. / Int. J. Biol. Chem. Sci. 14(3): 722-738, 2020

Tableau 7 : Hauteurs moyennes des cotonniers en fonction des systèmes de culture et des variétés de 2014 à 2018 à Sotuba.

\begin{tabular}{|c|c|c|c|c|c|c|c|c|c|c|c|c|c|c|c|}
\hline \multirow[b]{2}{*}{$\begin{array}{l}\text { Systèmes } \\
\text { de culture }\end{array}$} & \multicolumn{3}{|c|}{2014} & \multicolumn{3}{|c|}{2015} & \multicolumn{3}{|c|}{2016} & \multicolumn{3}{|c|}{2017} & \multicolumn{3}{|c|}{2018} \\
\hline & $\begin{array}{l}\text { NTA } \\
\text { MS334 }\end{array}$ & $\begin{array}{l}\text { BRS } \\
293\end{array}$ & Moy & $\begin{array}{l}\text { NTA } \\
\text { MS334 }\end{array}$ & $\begin{array}{l}\text { BRS } \\
293\end{array}$ & Moy & $\begin{array}{l}\text { NTA } \\
\text { MS334 }\end{array}$ & $\begin{array}{l}\text { BRS } \\
293\end{array}$ & Moy & $\begin{array}{l}\text { NTA } \\
\text { MS334 }\end{array}$ & $\begin{array}{l}\text { BRS } \\
293\end{array}$ & Moy & $\begin{array}{l}\text { NTA } \\
\text { MS334 }\end{array}$ & $\begin{array}{l}\text { BRS } \\
293\end{array}$ & Moy \\
\hline $\mathrm{T} 1$ & 112 & 104 & 112 & 142 & 136 & 139 & 120 & 102 & $111 \mathrm{~b}$ & 143 & 128 & 136 & 136 & 123 & 129 \\
\hline $\mathrm{T} 2$ & 135 & 128 & 131 & 136 & 133 & 134 & 125 & 123 & $124 \mathrm{a}$ & 150 & 130 & 140 & 135 & 131 & 133 \\
\hline $\mathrm{T} 3$ & 130 & 118 & 124 & 138 & 135 & 137 & 115 & 97 & $106 \mathrm{~b}$ & 149 & 134 & 141 & 134 & 126 & 130 \\
\hline $\mathrm{T} 4$ & 131 & 118 & 125 & 144 & 136 & 140 & 106 & 105 & $105 \mathrm{~b}$ & 143 & 133 & 138 & 133 & 124 & 128 \\
\hline T5 & 122 & 113 & 117 & 134 & 131 & 132 & 117 & 90 & $104 \mathrm{~b}$ & 134 & 123 & 128 & 128 & 123 & 126 \\
\hline T6 & 137 & 114 & 125 & 136 & 138 & 137 & 114 & 99 & $106 \mathrm{~b}$ & 144 & 130 & 137 & 127 & 123 & 125 \\
\hline Moyenne & $129 \mathrm{a}$ & $116 \mathrm{~b}$ & 122 & 138 & 135 & 137 & $116 \mathrm{a}$ & $103 \mathrm{~b}$ & 109 & $144 \mathrm{a}$ & $130 \mathrm{a}$ & 138 & $132 \mathrm{a}$ & $125 \mathrm{~b}$ & 129 \\
\hline $\begin{array}{l}\text { Probabilité } \\
\text { traitement }\end{array}$ & & & 0,064 & & & 0,582 & & & 0,009 & & & 0,107 & & & 0,324 \\
\hline $\begin{array}{l}\text { Probabilité } \\
\text { variété }\end{array}$ & & & 0,001 & & & 0,216 & & & 0,000 & & & 0,000 & & & 0,005 \\
\hline $\begin{array}{l}\text { Probabilité } \\
\text { interaction }\end{array}$ & & & 0,824 & & & 0,939 & & & 0,183 & & & 0,939 & & & 0,865 \\
\hline Ecart type & & & 9,19 & & & 2,12 & & & 0,11 & & & 0,08 & & & 0,07 \\
\hline CV (\%) & & & 9,80 & & & 6,80 & & & 10,06 & & & 6,07 & & & 5,75 \\
\hline
\end{tabular}

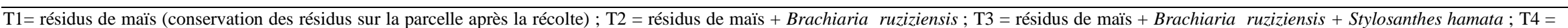

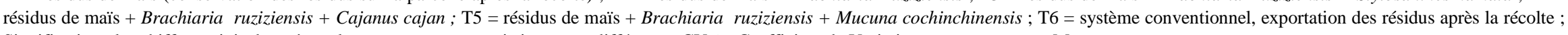
Signification : les chiffres suivis des mêmes lettres ne sont pas statistiquement différents ; CV\% : Coefficient de Variation en pourcentage ; Moy $=$ moyenne. 


\section{F. SISSOKO et al. / Int. J. Biol. Chem. Sci. 14(3): 722-738, 2020}

Tableau 8 : Nombre de capsules par plant en fonction des systèmes de culture et des variétés de 2014 à 2018 à Sotuba.

\begin{tabular}{|c|c|c|c|c|c|c|c|c|c|c|c|c|c|c|c|}
\hline \multirow[b]{2}{*}{$\begin{array}{l}\text { Systèmes } \\
\text { de culture }\end{array}$} & \multicolumn{3}{|c|}{2014} & \multicolumn{3}{|c|}{2015} & \multicolumn{3}{|c|}{2016} & \multicolumn{3}{|c|}{2017} & \multicolumn{3}{|c|}{2018} \\
\hline & $\begin{array}{l}\text { NTA } \\
\text { MS334 }\end{array}$ & $\begin{array}{l}\text { BRS } \\
293\end{array}$ & Moy & $\begin{array}{l}\text { NTA } \\
\text { MS334 }\end{array}$ & $\begin{array}{l}\text { BRS } \\
293\end{array}$ & Moy & $\begin{array}{l}\text { NTA } \\
\text { MS334 }\end{array}$ & BRS 293 & Moy & $\begin{array}{l}\text { NTA } \\
\text { MS334 }\end{array}$ & $\begin{array}{l}\text { BRS } \\
293\end{array}$ & Moy & $\begin{array}{l}\text { NTA } \\
\text { MS334 }\end{array}$ & $\begin{array}{l}\text { BRS } \\
293\end{array}$ & Moy \\
\hline $\mathrm{T} 1$ & 16,15 & 20,30 & 18,22 & 10.30 & 11.25 & 10,77 & 14,08 & 18,33 & 16,21 & 13,33 & 15,02 & 14,18 & 11,85 & 13,65 & 12,8 \\
\hline $\mathrm{T} 2$ & 14,55 & 21,55 & 18,05 & 9.70 & 14.35 & 12,02 & 14,75 & 16,75 & 15,75 & 13,15 & 16,75 & 14,95 & 12,65 & 14,60 & 13,6 \\
\hline $\mathrm{T} 3$ & 15,30 & 21,10 & 18,20 & 11.35 & 11.35 & 11,35 & 14,42 & 17,33 & 15,88 & 14,23 & 17,33 & 15,78 & 12,85 & 14,60 & 13,7 \\
\hline $\mathrm{T} 4$ & 17,05 & 21,35 & 19,20 & 13.50 & 13.60 & 13,55 & 14,00 & 17,00 & 15,50 & 13,26 & 17,00 & 15,13 & 12,50 & 13,45 & 13,0 \\
\hline T5 & 15,80 & 22,75 & 19,27 & 10.45 & 15.25 & 12,85 & 13,92 & 17,00 & 15,46 & 13,62 & 17,00 & 15,31 & 13,65 & 13,40 & 13,5 \\
\hline T6 & 15,05 & 22,35 & 18,70 & 10.75 & 12.75 & 11,75 & 13,33 & 17,42 & 15,38 & 13,03 & 15,05 & 14,04 & 12,85 & 13,60 & 13,2 \\
\hline Moyenne & $15,65 \mathrm{~b}$ & $21,57 \mathrm{a}$ & 18,61 & $11,0 \mathrm{~b}$ & 13,1 & 12,05 & $14,08 \mathrm{~b}$ & $17,31 \mathrm{a}$ & 15,69 & $13,43 \mathrm{~b}$ & $16.36 \mathrm{a}$ & 14,90 & $12,73 \mathrm{~b}$ & $13,88 \mathrm{a}$ & 13,30 \\
\hline $\begin{array}{l}\text { Probabilité } \\
\text { traitement }\end{array}$ & & & 0,944 & & & 0,127 & & & 0,931 & & & 0,148 & & & 0,439 \\
\hline $\begin{array}{l}\text { Probabilité } \\
\text { variété }\end{array}$ & & & 0,001 & & & 0,002 & & & 0,000 & & & 0,000 & & & 0,007 \\
\hline $\begin{array}{l}\text { Probabilité } \\
\text { interaction }\end{array}$ & & & 0,840 & & & 0,084 & & & 0,809 & & & 0,614 & & & 0,554 \\
\hline Ecart type & & & 2,21 & & & 1,05 & & & 1,75 & & & 1,44 & & & 1,15 \\
\hline $\mathrm{CV}(\%)$ & & & 8,60 & & & 1,40 & & & 11,13 & & & 9,64 & & & 8,61 \\
\hline
\end{tabular}


F. SISSOKO et al. / Int. J. Biol. Chem. Sci. 14(3): 722-738, 2020

Tableau 9 : Rendements obtenus en fonction des systèmes de culture et des variétés de 2014 à 2018 à Sotuba.

\begin{tabular}{|c|c|c|c|c|c|c|c|c|c|c|c|c|c|c|c|}
\hline \multirow[b]{2}{*}{$\begin{array}{c}\text { Système } \\
\text { de culture }\end{array}$} & \multicolumn{3}{|c|}{2014} & \multicolumn{3}{|c|}{2015} & \multicolumn{3}{|c|}{2016} & \multicolumn{3}{|c|}{2017} & \multicolumn{3}{|c|}{2018} \\
\hline & $\begin{array}{l}\text { NTA } \\
\text { MS334 }\end{array}$ & $\begin{array}{l}\text { BRS } \\
293\end{array}$ & Moy & $\begin{array}{l}\text { NTA } \\
\text { MS334 }\end{array}$ & $\begin{array}{l}\text { BRS } \\
293\end{array}$ & Moy & $\begin{array}{l}\text { NTA } \\
\text { MS334 }\end{array}$ & $\begin{array}{l}\text { BRS } \\
293\end{array}$ & Moy & $\begin{array}{l}\text { NTA } \\
\text { MS334 }\end{array}$ & $\begin{array}{l}\text { BRS } \\
293\end{array}$ & Moy & $\begin{array}{l}\text { NTA } \\
\text { MS334 }\end{array}$ & $\begin{array}{l}\text { BRS } \\
293\end{array}$ & Moy \\
\hline $\mathrm{T} 1$ & 2876 & 3536 & 3206 & 2531 & 2542 & 2536 & 2231 & 2700 & 2466 & 1756 & 2436 & 2096 & 1690 & 2102 & 1896 \\
\hline $\mathrm{T} 2$ & 3148 & 3993 & 3571 & 2302 & 2656 & 2479 & 2294 & 2832 & 2563 & 1746 & 2339 & 2043 & 1742 & 1671 & 1707 \\
\hline $\mathrm{T} 3$ & 3073 & 3773 & 3423 & 2531 & 2667 & 2599 & 2057 & 2611 & 2334 & 1654 & 2183 & 1919 & 1781 & 2134 & 1957 \\
\hline $\mathrm{T} 4$ & 3160 & 3782 & 3471 & 2396 & 2865 & 2630 & 2083 & 2479 & 2281 & 1861 & 2436 & 2148 & 1605 & 1927 & 1766 \\
\hline T5 & 3322 & 4230 & 3776 & 2125 & 2677 & 2401 & 2004 & 2294 & 2149 & 1838 & 2528 & 2183 & 1494 & 1900 & 1697 \\
\hline T6 & 3183 & 3414 & 3299 & 2281 & 2656 & 2469 & 2041 & 2442 & 2242 & 1792 & 2505 & 2148 & 1665 & 1746 & 1706 \\
\hline Moyenne & $3127 b$ & $3788 \mathrm{a}$ & 3458 & $2361 \mathrm{~b}$ & $2677 \mathrm{a}$ & 2519 & $2118 b$ & $2560 \mathrm{a}$ & 2339 & $1775 \mathrm{~b}$ & $2404 \mathrm{a}$ & 2089 & $1663 \mathrm{~b}$ & $1914 a$ & 1788 \\
\hline $\begin{array}{l}\text { Probabilité } \\
\text { traitement }\end{array}$ & & & 0,112 & & & 0,677 & & & 0,690 & & & 0,359 & & & 0,526 \\
\hline $\begin{array}{l}\text { Probabilité } \\
\text { variété }\end{array}$ & & & 0,001 & & & 0,001 & & & 0,008 & & & 0,000 & & & 0,000 \\
\hline $\begin{array}{l}\text { Probabilité } \\
\text { interaction }\end{array}$ & & & 0,645 & & & 0,497 & & & 0,990 & & & 0,818 & & & 0,137 \\
\hline Ecart type & & & 85 & & & 223 & & & 547 & & & 251 & & & 340 \\
\hline $\mathrm{CV}(\%)$ & & & 11,8 & & & 12,2 & & & 23,38 & & & 12,02 & & & 18,98 \\
\hline
\end{tabular}

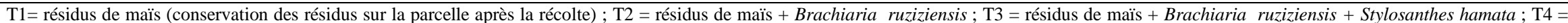

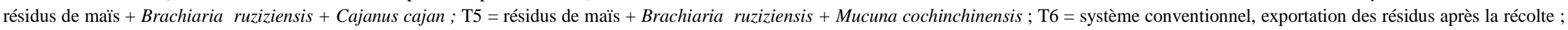
Signification : les chiffres suivis des mêmes lettres ne sont pas statistiquement différents ; CV\% : Coefficient de Variation en pourcentage $;$ Moy $=$ moyenne. 


\section{DISCUSSION}

En zone soudano-sahélienne, le cumul annuel de la pluviométrie ne semble pas être un bon indicateur de la disponibilité de l'eau pour les cultures, car sa distribution spatiotemporelle est très souvent mauvaise. L'analyse de la pluviométrie du mois de juin, le plus propice au semis du cotonnier, a montré une très grande variabilité entre les décades de la même année, avec souvent des décades sans pluie. La date optimale de semis du cotonnier se situe dans la deuxième décade du mois de juin à Sotuba. Si les quantités de pluies enregistrées pendant cette période sont faibles, les semis sont réalisés dans la troisième décade du mois de juin. Quand la pluie est bien répartie, le cotonnier a besoin d'environ 600 $\mathrm{mm}$ d'eau durant son cycle de culture (Cetin et Bilgel, 2002).

Les valeurs obtenues après l'analyse des échantillons de sol sont supposées être les plus faibles qu'on puisse observer au cours de l'année selon les travaux réalisés par Sissoko (2009). Les valeurs de pH eau supérieurs à 6 dans ce sol, n'ont pas d'effet négatif sur la vie microbienne et sur les formes chimiques des éléments nutritifs du sol selon Bertrand et Gigou (2000). Le pH eau n'est certainement pas le seul paramètre permettant de bien apprécier des propriétés du sol. D'autres paramètres comme la Capacités d'Echange Cationique, les bases échangeables, la teneur en matière organique etc. peuvent fournir des informations intéressantes. Les paramètres évalués $(\mathrm{pH}$, azote, phosphore total et assimilable, le potassium, le calcium, le magnésium, et la capacité d'échange cationique) montrent que les sols de la zone d'étude sont pauvres.
Les difficultés de germination sont très souvent liées à l'insuffisance d'humidité dans le sol. La conservation de la biomasse en couverture sur le sol permet de mieux conserver l'humidité (Scopel et al., 2004 ; Sissoko, 2009 ; Dugué et al., 2015 ; Ouattara et al., 2018). La différence en nombre de jours entre un semis réalisé sous couverture végétale et un semis en système conventionnel peut atteindre 4 jours. Cette vitesse à la levée peut impacter la densité de poquets et de plants par hectare.

Certains auteurs ont montré qu'il n'y a pas de relation précise entre le nombre de poquets à la récolte et le rendement obtenu dans les différents systèmes de culture. Entre 30000 et 40000 poquets.ha $^{-1}$, les rendements varient fortement entre 1 et 2,3 t.ha ${ }^{-1}$ en fonction des systèmes de culture. Bednarz et al. (2000) ont montré que le rendement n'est pas influencé par des densités variantes entre 2,5 à 23 poquets $/ \mathrm{m}^{2}$. Rapidel et al. (2006) ont obtenu des rendements statistiquement équivalents avec des densités variant entre 34000 et 48300 plants.ha-1 ${ }^{-1}$ Ceci s'explique par l'ajustement du pourcentage des capsules sur les différentes positions qui annule l'effet de la forte densité. Sekloka et al. (2016) ont trouvé qu'à faible densité, les cotonniers sont plus florifères et retiennent mieux leurs capsules, les nombres moyens de sites fructifères par plants et les taux de rétention sont plus élevés à 42000 plants.ha-1 qu'à 125000 et 167000 plants.ha ${ }^{-1}$. A l'inverse Smith et al. (1979) ont montré que la production de coton graine est significativement différente pour des fortes densités (170 000 poquets.ha $\left.{ }^{-1}\right)$ comparés aux faibles densités (34000 poquets.ha-1 ${ }^{-1}$ ).

Les systèmes de culture n'ont pas eu d'effet sur la hauteur des cotonniers à récolte. 
Au Burkina Faso, Sourgou (2018) a obtenu les mêmes résultats sur deux sites d'étude à Houndé et à Bondokuy. Cependant, à Tiakaré et Kouaré, il a observé les meilleures hauteurs avec le coton en culture conventionnelle. Une différence significative a été constatée entre les deux variétés. La BRS 293 est généralement plus courte de taille.

Le nombre de capsules par plants a été statistiquement équivalent dans les parcelles des différents systèmes de culture. Ce résultat est confirmé par les travaux de Sourgou (2018) qui n'a lui aussi pas obtenu de différence significative entre le nombre de capsules par hectare dans les parcelles en SCV comparées aux parcelles du système conventionnel.

En fonction de l'architecture du cotonnier, le nombre de capsules par plant peut varier. Le meilleur nombre de capsules par plant a été obtenu sur la variété BRS 293 qui a une production plus groupée sur les premières positions des branches végétatives.

Les rendements en coton graine n'ont pas été influencés par les systèmes de culture étudiés. Des observations similaires ont été faites par Swanepoel et al. (2017) qui ont montré que les études de courtes durées ne permettent pas de mettre en évidence des améliorations significatives de la teneur en matière organique du sol et des rendements en agriculture de conservation. Le semis sous couverture végétale est présenté comme la panacée aux problèmes de faible productivité agricole et de dégradation des sols en Afrique subsaharienne (Giller et al., 2009). Cependant, il y a des préalables à la réussite de cette innovation. Le respect de l'itinéraire technique a permis d'avoir des rendements deux fois supérieurs au rendement moyen de la zone de production du coton au Mali qui est de $984 \mathrm{~kg}$. ha $^{-1}$ (Traoré, 2018).

\section{Conclusion}

Il ressort clairement de ces travaux, les effets positifs du semis direct sous couverture végétale sur les différents paramètres agronomiques des deux variétés de cotonnier utilisées dans cette expérimentation. Le semis direct sous couverture végétale a permis une installation rapide des cotonniers avec des pluies de faibles quantités. Le nombre de plants levés a été amélioré dans les systèmes de semis direct (SCV) par rapport au système conventionnel. Le semis direct sous couverture végétal n'a pas affecté la croissance des cotonniers qui a été normale dans l'ensemble. Aucune différence significative n'a été observée entre les rendements obtenus dans les systèmes de culture. Cela dénote que les systèmes de cultures en SCV permettent de bien produire le coton et d'économiser le labour. Par conséquent, le semis direct sous couverture végétale pourrait être une alternative pour la culture du cotonnier, notamment dans la zone semi-aride où la pluviométrie est de plus en plus erratique avec une grande variabilité spatio-temporelle.

\section{CONTRIBUTIONS DES AUTEURS}

Les co-auteurs Sidiki DIARRA et Mamadou TRAORE ont mis en place les expérimentations. Ils ont également suivi, collecté et saisi toutes les données de cet article. Fagaye SISSOKO a analysé, interprété et rédigé l'article.

\section{REMERCIEMENTS}

Les auteurs remercient les chercheurs de l'Embrapa/Brésil et du programme coton de 
l'IER pour la fourniture du matériel végétal et les recommandations pour le suivi du parasitisme.

\section{REFERENCES}

Bationo A, Kihara J, Vanlauwe B, Waswa B, Kimetu J. 2007. Soil organic carbon dynamics, functions and management in West African agro-ecosystems. Agric. Syst., $\quad$ 94: 13-25. DOI: 10.1016/j.agsy.2005.08.011

Baudron F, Mwanza HM, Triomphe B, Bwalya M. 2007. Conservation Agriculture in Zambia: A Case Study of Southern Province. African Conservation Tillage Network, Centre de Coopération Internationale de Recherche Agronomique pour le Development, Food and Agricultural Organization of the United Nations, Nairobi.

Bertrand R, Gigou J. 2000. La Fertilité des Sols Tropicaux. Maisonneuve et Larose: Paris.

Bednarz CW, Bridges DC, Brown SM. 2000. Analyse de la stabilité du rendement du coton à travers les densités de population. Agron. J. 92 : 128-135. DOI : 10.2134/agronj2000.921128x.

Camara M. 2015. Atouts et limites de la filière coton au Mali. Thèse de Doctorat, Toulon, p. 321.

Cetin O, Bilgel L. 2002. Effets of different irrigation methods on shedding and yield of cotton. Agricultural Water Management, $\quad \mathbf{5 4}(1)$ : 1-15. DOI: https://doi.org/10.1016/S03783774(01)00138-X

Dugué P, Djamen NP, Faure G, Le Gal PY. 2015. Dynamiques d'adoption de l'agriculture conservation dans les exploitations familiales : de la technique aux processus d'innovation. Cah. Agric., 24: 60-68.

DOI: http://dx.doi.org/10.1684/agr.2015.0748

Deveze JC, Halley des Fontaines D. 2005. Débats, Echanges, Réflexions en Réseaux Développement Rural au Sud. Document AFD, Paris.

Fairhurst T. 2015. Manuel de Gestion Intégrée de la Fertilité des Sols. Consortium Africain pour la Santé des Sols, Nairobi.

Fleury M. 2016. Agriculture itinérante sur brûlis (AIB) et plantes cultivées sur le haut Maroni : étude comparée chez les Aluku et les Wayana en Guyane Française, Brasil. Boletim do Museu Paraense Emílio Goeldi. Ciências Humanas, 11(2): 431-465. DOI:

http://dx.doi.org/10.1590/1981.81222016 000200006

Gowing JW, Palmer M. 2008. Sustainable agricultural development in sub- Saharan Africa: the case for a paradigm shift in land husbandry. Soil Use Manage, 24 (1), 92-99.

DOI: https://doi.org/10.1111/j.1475-

2743.2007.00137.x

Giller KE, Andersson JA, Corbeels M, Kirkegaard J, Mortensen D, Erenstein O, Vanlauwe B. 2015. Beyond conservation agriculture. Front. Plant Sci., 6: 870. DOI:

https://doi.org/10.3389/fpls.2015.00870

Giller KE, Witter E, Corbeels M, Tittonelle P. 2009. Conservation agriculture and smallholder farming in Africa: The heretics' view. Field Crops Research, 114(1): 23-34. DOI: https://doi.org/10.1016/j.fcr.2009.06.017 
Kouressy M, Sultan B, Vaksmann M, Belières JF, Claessens L, Dingkuhn M, Témé N. 2019. Climate Change and Cereal Production Evolution Trend in the Sahel: Case Study in Mali from 1951 to 2010. Sustainable Agriculture Research, 8(2): 68-89.

DOI:

https://doi.org/10.5539/sar.v8n2p68

Naudin K, Gozé E, Balarabé O, Giller KE, Scopel E. 2010 - Impact of no-tillage and mulching practices on cotton production in North Cameroon: a multi-locational on-farm assessment. Soil and Tillage Research, $\quad$ 108(1-2): 68-76. DOI: 10.1016/j.still.2010.03.002

Naudin K, Bruelle G, Salgado P, Penot E, Scopel E, Lubbers M, De Ridder N, Giller KE. 2015. Trade-offs around the use of biomass for livestock feed and soil cover in dairy farms in the Alaotra lake region of Madagascar. Agricultural Systems, 134: 36-47. DOI: https://doi.org/10.1016/j.agsy.2014.03.00 3

Odru M. 2013. Flux de biomasse et renouvellement de la fertilité des sols à l'échelle du terroir. Etude de cas d'un terroir villageois sereer au Sénégal. Mémoire de fin d'études. Ecole Supérieure d'Agro-Développement International. Dakar, Sénégal, p. 109.

Ouattara B, Coulibaly K, Kohio E, Doumbia S, Ouédraogo S, Nacro HB. 2018. Effets du Système de Culture sous couverture Végétale (SCV) sur les flux hydriques d'un sol ferrugineux à l'ouest du Burkina Faso. Int. J. Biol. Chem. Sci., 12(4): 17701783.

DOI:

https://dx.doi.org/10.4314/ijbcs.v12i4.20
Rapidel B, Defeche C, Traore B, Lançon J, Wery J. 2006. In-field development of a conceptual crop functioning and management model: A case study on cotton in southern Mali. European Journal of Agronomy, 24: 304-315. DOI:

https://doi.org/10.1016/j.eja.2005.10.012

Rockstrom J, Kaumbutho P, Mwalley J, Nzabi AW, Temesgen M, Mawenya L, Barron J, Mutua J, Damgaard-Larsen S. 2008. Conservation farming strategies in East and Southern Africa: yields and rain water productivity from on-farm action research. Soil Till. Res., 103: 23-32. DOI :

https://doi.org/10.1016/j.still.2008.09.01 3

Sekloka E, Lançon J, Zinsou VA, Thomas G. 2016. Influence des conditions de culture sur la production de capsules chez le cotonnier (Gosssypium hirsutum L.) en conditions de culture pluviale au Bénin. Biotechnol. Agron. Soc. Environ., 20(2): 161-170. DOI: $10.25518 / 1780$ 4507.12904

Scopel E, Macena F, Corbeels M, Affholder F, Maraux F. 2004. Modeling crop residue mulching effects on water use and production of maize under semi-arid and humid tropical conditions. Agronomy, 24: 383-395. DOI: 10.1051/agro: 2004029

Smith CW, Waddle BA, Ramey HH Jr. 1979. Plant Spacings with irrigated Cotton. Agronomy Journal, 71 : 858-860. DOI : https://doi.org/10.2134/agronj1979

Sissoko F. 2009. Analyse des flux d'eau dans les systèmes de culture sous couverture végétale en zone Soudano sahélienne : Cas du coton semé après une culture de 
Sorgho/Brachiaria au Sud du Mali. Thèse de doctorat de SupAgro, Montpellier, p. 169.

Sissoko F, Affholder F, Autfray P, Wery J, Rapidel B. 2013. Wet years and farmers' practices may offset the benefits of residue retention on runoff and yield in cotton fields in the Sudan-Sahelian zone. Agricultural Water Management, 119: 89-99.

DOI: http://dx.doi.org/10.1016/j.agwat.2012.1 2.012

Sissoko F, Coulibaly D, Cissé O, Dugué P. 2015. Évaluation de l'arrière effet de la culture du coton sur la production céréalière en zone cotonnière du Mali. In AGRAR-2013 $1^{e}$ Conférence de la Recherche Africaine sur l'Agriculture, l'Alimentation et la Nutrition. Presses Agronomiques de Gembloux ; 149-159.

Sourgou L. 2018. Effets du semis direct sous couverture végétale (SCV) sur les rendements du maïs et du cotonnier dans les zones cotonnières du Burkina Faso. Mémoire Université Nazi Boni, BoboDioulasso, p. 77.

Swanepoel CM, Swanepoel LH, Smith HJ. 2017. A review of conservation agriculture research in South Africa. South African Journal of Plant and Soil, $1-10$.

DOI: 10.1080/0571862.2017.1390615

Traoré M. 2018. Rapport bilan de fin campagne 2018/2019 de la Compagnie Malienne pour le Développement des Textiles du Mali, p. 61.

Turnel MS, Speratti A, Baudrin F, Verhulst N, Govaerts B. 2015. Crop residue management and soil health: $\mathrm{S}$ systems analysis. Agricultural Systems, $134: 6-$ 16. DOI : https://doi.org/10.1016/jagsy2014.05.009. 